Cita bibliográfica: Fernández Borrero, M. A., Relinque Medina, F. y Martí García, S. (2017). Percepciones para la intervención social desde la competencia intercultural de profesionales [Perceptions for social intervention from professionals intercultural competence]. Alternativas. Cuadernos de Trabajo Social, 24, 91-118. https://doi.org/10.14198/ ALTERN2017.24.06

\title{
PERCEPCIONES PARA LA INTERVENCIÓN SOCIAL DESDE LA COMPETENCIA INTERCULTURAL DE PROFESIONALES
}

\section{PERCEPTIONS FOR SOCIAL INTERVENTION FROM PROFESSIONALS' INTERCULTURAL COMPETENCE}

\author{
MANUELA-ÁNGELA FERNÁNDEZ BORRERO \\ Departamento de Sociología, Trabajo Social y Salud Publica de la Universidad de Huelva. \\ Facultad de Trabajo Social \\ Manuela.fernandez@dstso.uhu.es \\ FERNANDO RELINQUE-MEDINA \\ Departamento de Sociología, Trabajo Social y Salud Publica de la Universidad de Huelva. \\ Facultad de Trabajo Social \\ Fernando.relinque@pi.uhu.es \\ SUSANA MARTÍ-GARCÍA \\ Departamento de Sociología, Trabajo Social y Salud Publica de la Universidad de Huelva. \\ Facultad de Trabajo Social \\ Susana.marti@dstso.uhu.es
}

\begin{abstract}
Resumen
En este trabajo mostramos un análisis de las respuestas dadas por profesionales de los servicios sociales comunitarios a supuestos prácticos relacionados con la intervención profesional en contextos de diversidad cultural. Se pretende conocer si las respuestas a estos supuestos se relacionan con características de la intervención profesional, rasgos personales y con variables como la Competencia Intercultural y la Sensibilidad Intercultural. Han participado 253 profesionales (psicólogos, trabajadores sociales y educadores sociales). Se han realizado análisis de varianza, asociaciones con contingencia, correlaciones y Análisis Factorial de Correspondencias. Los resultados muestran que la profesión no influye en dar una respuesta adecuada a los supuestos. En cambio, tener formación en diversidad y contacto con personas de origen extranjero presentan asociaciones significativas con responder adecuadamente a ciertos supuestos. Hemos obtenido una tipología de cuatro clases diferenciadas según las respuestas, dos positivas respecto a la resolución de los supuestos y su vinculación con la competencia intercultural y dos con carácter negativo.
\end{abstract}

Palabras clave: competencia intercultural, servicios sociales, análisis de casos, intervención social, sensibilidad intercultural

\begin{abstract}
This work analysizes the responses of professionals to community social services practical cases related to professional intervention in contexts of cultural diversity. We want to know if these responses relate to the characteristics of professional intervention, personal traits and variables such as Intercultural Competence and Intercultural Sensitivity. 253 professionals have participated (psychologists, social workers and community workers). We have conducted variance analysis, measures of associations for contingency tables, correlations and Factorial Correspondence Analysis. The results show that the profession is not related to an appropriate response to the cases. Instead, training on diversity and contact with people of foreign origin has significant associations with appropriately response to certain assumptions. We have obtained a typology of four distinct classes according to responses; two positive classes regarding case resolution and their connection with intercultural competence, and two negative classes.
\end{abstract}

Keywords: intercultural competence, social services, case studies, social intervention, social 


\section{Extended abstract}

\section{Introduction}

Modern societies are culturally diverse and plural where different cultural groups living together within a shared social and political framework (Skelton y Allen, 1999). Beyond the diverse conformation of society, these flows and migration processes have also had a considerable impact on public services, especially in the medical field, which demands effective adjustments to different users and their needs. Providing culturally appropriate services is important for the development of societies and is one of the major challenges to be met by public services and professionals of social intervention (Martinez, Martinez and Calzado 2006).

In order to understand Spanish reality, it is necessary to know that access to public welfare services by foreign population is granted by Royal Decree $844 / 2013$, amending the regulations of the Organic Law 4/2000 on the rights and freedoms of foreigners in Spain. However, each region implements it in a particular way because, at the policy level, Autonomous Communities have transferred competences in social services (Andalusia - law 2/1988 of 4 April).

It is important to consider the characteristics of good intercultural practices in the provision of services based on universal access, subject active participation, duration in time, ongoing assessment and monitoring and facilitating social cohesion (Vázquez, Fernández \& Fernández-Borero, 2010:305).

\section{Professionals of Community Social Services in contexts of cultural diversity}

We consider it important that professionals ask themselves about the cultural conceptions from which they analyze the situations and demands that reach community social services, as much as about the evaluation, diagnosis and approaches to intervention strategies as they incorporate the ethical and professional notions involved and and necessary for each case.

In this paper, the starting point are the dimensions of the IC assumed as relevant for professionals in community social services (Martinez et al., 2006) with a number of practical scenarios developed by our research team.

The construction of these practical cases is the result of the combination of several elements: the training and professional practice of Social Work and Psychology of the members of the research team; the literature reviewed; and the joint and participative consultation and construction with professionals of Community Social Services. They are therefore, real cases adapted for study.

We aim to answer questions such as: is responding appropriately to cases associated with having high levels of IC?; what dimensions establish associations, or which ones gain more relevance in providing a higher number of correct answers to each of the scenarios presented?, Are there differences due to the personal characteristics of participants that may require appropriate responses to cases? The aim of this study was to determine the associations between the answers to the practical cases with professional features, the self-assigned IC level and IS. Also, we try to know if differences arise from professional practice, having different levels of IC or different socio-demographic characteristics (this is not clear, which are the terms the differences between which are to be confirmed?) responding adequately to the practical cases presented. Our last goal is drawing basic profiles depending on the variables under study.

\section{Methods}

\section{Participants}

This work has involved 253 individuals from the different professions that constitute the core team in Andalusian community social services Social Work, Psychology and Social Education.

\section{Instrument}

The instrument contains different scales:

Sociodemographic questions

A scale with nine practical cases of professional performance that must be assessed as right or wrong

A five-item Likert scale to assess the dimensions of self-attributed IC described by Martínez et al. (2006)

Another five-point Likert scale on issues of professional intervention with persons of foreign origin (Martínez et al 2006)

We also include the Spanish translation (Vila, 2005) of Chen and Starosta IS Scale (2000), with 24 items and five dimensions: involvement, respect, trust, enjoyment and attention.

\section{Analysis strategy}

We conducted an initial basic characterization of the participants in the different scales.

The main analyses were based on practical cases and related skills; we calculated correlations (Pearson $\chi 2$ ), associations, mean comparisons and variance analysis. Cohen's d (Cohen 1988) was used to calculate effect sizes. Finally, we carried ot an a Multiple Correspondence Factor Analysis (CFA).

We used the statistical software SPSS 20.0. The Anaconda software belonging to the group Catalyse tools was also used, combining factorial correspondence analysis and ascending hierarchical classification. 


\section{Results}

The accuracy rate of the cases was variable, ranging from $15,8 \%$ (case three) to $94.3 \%$ (case four), with an average level of correct answers of 4 out of the total 9 cases presented. 42.1 of the participants are at a low level of correct responses (three or less), 48.9 percent at a medium level (four to six right answers) and the remaining 9.1 percent, at a high level (seven or more properly answered cases).

The profession does not produce significant differences in the average number of cases answered correctly. Having training in cultural diversity is significantly associated with responding appropriately to the sixth case, related to preserving the professional's personal information from users and reflecting the ability to socialize and the proper management of social distance.

\section{Results on intercultural competece}

In general, terms, the overall IC is high, particularly at the conscious level, with a remarkably higher level in psychologists. In the dimensions of awareness and motivation to find out the highest competence (unconscious), social educators stand out against other professionals. In the other dimensions (knowledge, skills and encounter), the three professions show similar levels. There are no statistically significant differences between professions.

Globally IC is associated with the second case (refusal to handshake the professional with his/her left hand), so that the difference between responding properly or not occurs at the highest levels of competence; with the highest level (unconscious competence) giving correct answers; and somewhat lower levels-although high (conscious competence)-responding erroneously. Association also occurs with the eighth case, establishing the association between the level of intercultural incompetence and inappropriate response.

In matters relating to the intervention, diversity management skills stand out positively, while the lower mean shows in self-awareness of one's own biases and prejudices.

Moreover, IS is quite high ( $\mathrm{M}=4.1, \mathrm{SD}=0.44)$, with a significantly high respect for cultural differences. The total correct responses to cases not correlated with scores in global IS or in each of its dimensions.

\section{Typological analysis of results}

In this analysis, each of the axes is determined largely by a variable or a set of them. Levels of IC competence and incompetence dimensions are distributed along axis 1 , but it is also associated with IS levels. Axis 2 is related in a more direct way with the practical cases.
Aspects of professional intervention are distributed along axis 3. The results show a total of four classes.

Class 1 includes most psychologists. They are individuals with knowledge of other languages and daily contact with foreigners. They have skills in intervention and perceive the cultural diversity of the people and situations in which they work. They also know how to implement projects in these contexts and have knowledge about intercultural praxis. In addition, this class combines the most appropriate answers to cases.

Class 2. It is characterized by the highest level of IC competence in all dimensions (except for motivation). Provides a proper response to three cases (second, fourth and ninth), as well as the presence of high IS levels

Class 3 is characterized by neutrality in aspects of intervention in intercultural contexts. They have been trained in diversity at a level of incompetence in the dimension of intercultural awareness, but of competence in the motivational dimension of IC.

Class 4 shows IC levels of incompetence and IS medium levels. They are far from giving the correct answers to practical cases. In addition, they have no contact with foreigners and cannot speak other languages.

\section{Discussion and conclusions}

The results reveal some significant associations between responses to practical cases and the key variables of the study. Regarding characterization, no differences occur in the number of cases adequately answered by the different professions.

There are other matters of personal characterization that stand out, such as having training on cultural diversity, and contact with people of foreign origin. Having a certain level of IC is associated, in some cases, to successful professional interventions.

Adequate responses to a high number of cases are associated to professionals with higher intercultural awareness and a greater motivation for intercultural encounters.

The classes extracted revealed the existence of key issues in providing appropriate responses to cases that relate with high IC levels in the different dimensions considered as well as high IS levels that render key aspects for the development of professional practice in contexts of cultural diversity.

These issues can and should be dealt with the professionals of Community Social Services, including all the professions present in them, since no significant differences were found depending on profession. Training proposals can be developed to improve and tailor services and professional IC itself. 


\section{Introducción}

Los movimientos migratorios han sido muy relevantes para la construcción y conformación de la diversidad sociocultural. Han adquirido una gran importancia en Europa y en España en las últimas décadas del siglo XX y primera década del siglo XXI.

Las sociedades actuales son culturalmente diversas y plurales donde diferentes grupos culturales viven juntos dentro de un marco social y político compartido, como afirman Skelton y Allen (1999). Estos flujos y procesos migratorios han tenido también un considerable impacto en los servicios públicos, destacando el ámbito socio-sanitario, que demanda reajustes que permitan dar respuestas eficaces a los diferentes tipos de usuarios y necesidades. Disponer de servicios culturalmente adaptados es importante para el desarrollo de las sociedades y es a la vez, según Martínez, Martínez y Calzado (2006), uno de los principales retos a los que tienen que enfrentarse los servicios públicos y los profesionales de la intervención social.

Para entender la realidad española es necesario saber que el acceso a los servicios públicos de bienestar por parte de población extranjera queda reconocido por el Real Decreto 844/2013 por el que se modifica el reglamento de la Ley Orgánica 4/2000 sobre derechos y libertades de los extranjeros en España, aunque cada comunidad autónoma lo establece de un modo concreto dado que, a nivel normativo, son las Comunidades autónomas las que tienen transferidas las competencias en servicios sociales (la Ley 9/2016, de 27 de diciembre, de Servicios Sociales de Andalucía.).

Dentro de estos servicios sociales existen dos niveles: especializados, dirigidos a sectores particulares de población, y comunitarios, dirigidos a toda la población, siendo el primer nivel de atención y protección social.

Cuentan con una oferta específica de prestaciones sociales, un ámbito específico que es el de las necesidades sociales, y unos principios filosóficos que son: responsabilidad pública, solidaridad, igualdad y universalidad, participación ciudadana, descentralización, planificación/coordinación, interdisciplinariedad, normalización, globalidad y prevención. Sus funciones más importantes son adaptar los programas al contexto social, promover la participación, realizar acciones integrales y ser puerta de entrada al sistema.

El $6 \%$ del total de las personas usuarias de estos servicios son extranjeras (según datos de 2011 disponibles para Andalucía), siendo servicios referentes de primera entrada a otros sistemas sociales y dinámicas de inserción para muchas personas extranjeras; por este motivo, creemos que resulta necesaria 
la revisión de las respuestas y prácticas que se están desarrollando a la luz de la competencia intercultural (CI) de los profesionales de estos servicios.

\section{Competencia Intercultural (CI) y Servicios Sociales Comunitarios}

La noción de CI se entiende como la capacidad de dar respuestas satisfactorias en procesos interculturales incorporando comportamientos, actitudes y conocimientos que ayuden a la interacción social. En el marco de los servicios sociales, además de esta dimensión interactiva, la CI también implica el desarrollo de políticas institucionales que permitan a los profesionales ser eficaces y efectivos en situaciones de diversidad cultural. Se trata de ir más allá de una visión comprensiva de la diversidad hacia una efectiva praxis profesional intercultural. Se trataría de superar progresivamente el enfoque únicamente etic (desprovisto de cualquier intento de descubrir el significado que los agentes involucrados le dan a su realidad y acciones, con un punto de vista externo), incluyendo la perspectiva emic (que presenta la perspectiva interna al desglosar la interpretación del significado, con sus reglas y categorías, como el conocimiento sociocultural que rige y es común para un grupo o personas). Esto conduce a lo que Berry, Pootinga, Segall y Dasen. (2002: 291) denominan como «etics derivados» con gran validez intercultural, o considerando un enfoque etic flexible, entendido por Dumas et al. (1999: 182) como asumir una visión etic en la concepción pero siendo consciente y considerando la perspectiva emic existente. Es importante tener en consideración las características de las buenas prácticas interculturales en la prestación de servicios basada en el acceso universal, la participación activa de los sujetos, el mantenimiento en el tiempo, la evaluación y monitorización continua y el favorecimiento de la cohesión social (Vázquez, Fernández y Fernández-Borrero, 2010: 305).

En la lógica de la atención de los servicios culturalmente adaptados y competentes algunos aspectos básicos a considerar recogidos en la literatura son: el uso apropiado del idioma y el lenguaje, asegurando que sea comprensible y favoreciendo la capacidad de respuesta y equilibrio en las interacciones; el conocimiento de los valores y creencias presentes en la relación profesional, como puede ser el hecho de trabajar con el miedo a las consecuencias legales y burocráticas de minorías culturales; conocer las múltiples situaciones posibles derivadas de los procesos migratorios, así como las expectativas existentes sobre la relación profesional a establecer y servicios a recibir.

La CI se manifiesta cuando los profesionales entienden, aprecian, reconocen y respetan las diferencias, siendo capaces de ajustar sus prácticas y 
organizaciones al desarrollo de intervenciones efectivas en contextos de diversidad (Dana y Allen, 2008, Balcazar, Suarez-Balcazar y Willis, 2010). Se caracteriza, según Kohli, Huber y Faul (2010), por la aceptación y respeto por las diferencias, la consideración de las dinámicas diferenciales de funcionamiento, la expansión del conocimiento cultural y variedad de adaptaciones de los modelos de ayuda e intervención que permitan proveer servicios apropiados. Estos modelos pueden estar basados en la nueva lógica ecológico-sistémica del trabajo social que, según Ungar (2002), mantiene que la existencia de la diversidad social y cultural es un potencial para que el trabajo social genere nuevas soluciones para los problemas a los que se enfrenta, permitiendo emerger nuevos cambios profesionales y personales.

Los profesionales de los Servicios Sociales Comunitarios en contextos de diversidad cultural

Consideramos relevante que los profesionales se planteen desde qué concepciones culturales analizan las situaciones y demandas que llegan a los servicios sociales comunitarios, así como la evaluación, el diagnóstico y el planteamiento de la (s) estrategia(s) de intervención incorporando las nociones éticas y profesionales implícitas y necesarias en cada caso. Se trata de asumir el enfoque intercultural para ir más allá del reconocimiento de la diferencia del otro hasta lograr relaciones culturalmente más simétricas. Esta misma lógica conforma algunos de los principios deontológicos básicos de las profesiones fundamentales de los equipos base de los servicios sociales comunitarios: trabajo social, educación social y psicología. En este sentido, Garran y Werkmeister (2013) realizan una reflexión basada en los estándares establecidos en el año 2001 por la Asociación Nacional de Trabajadores Sociales de Estados Unidos, sobre la práctica culturalmente competente en un marco de compromiso profesional por la diversidad y la justicia social, y bajo la lógica de la interseccionalidad que implica comprender la diversidad cultural como un contexto más amplio de interconexiones de diversidades (género, étnica, sexual...), de influencias de poder, posicionamientos y elementos de fuerzas socio-político-estructurales que existen en cada sociedad. Implica tener una visión de la persona en cuanto a sus características individuales y sus circunstancias vitales (grupos a los que pertenece, elementos familiares, etc.), comprendiendo además que la cultura es un ingrediente de toda acción y persona en cualquier situación.

Los Standards for Cultural Competence in Social Work Practice (NASW, 2001) reflejan un avance del compromiso deontológico con la obligación 
profesional de prestar servicios culturalmente competentes, respondiendo con eficacia y bajo dos principios fundamentales: el respeto y el valor de las personas. A modo de decálogo, estos estándares recogen: 1) el desarrollo profesional de acuerdo con los valores, la ética y las normas de la profesión; 2) la comprensión de los propios valores apreciando la importancia de las identidades multiculturales; 3 ) disponer de conocimiento y la comprensión especializada sobre los elementos de las diferentes culturas; 4) disponer de habilidades interculturales, utilizando el marco metodológico y técnicas más apropiadas que reflejen una adecuada comprensión de la función de la cultura en el proceso de ayuda; 5) poner en práctica un buen servicio y asesoramiento útil; 6) desarrollar empoderamiento; 7) abogar por la diversidad y enriquecimiento dentro de la profesión y en los diferentes servicios y proyectos; 8) promover y participar en los programas de educación y capacitación que ayudan a avanzar en la competencia cultural; 9) realizar un esfuerzo por proporcionar información, referencias y servicios en el lenguaje apropiado para la persona, aunque ello requiera el uso de mediadores y/o intérpretes; y 10) desarrollar liderazgo intercultural siendo capaces de comunicar información sobre los grupos y comunidades a otros profesionales.

Estos estándares guardan una estrecha relación con las bases deontológicas profesionales. A nivel internacional, el Código de Ética de la International Federation of Social Workers (IFSW, 2004) y de la International Association of Schools of Social Work (IASSW, 2004), recogen que los trabajadores sociales deben reconocer y respetar la diversidad étnica y cultural de las sociedades con las que trabajan, teniendo en cuenta las diferencias individuales, familiares, grupales y comunitarias. El Código Deontológico del Trabajo Social en España (Consejo General del Trabajo Social, 2012) establece la igualdad como principio básico que garantiza los mismos derechos y deberes compatibles con las peculiaridades y diferencias (art. 7), así como el principio de individualización que recoge la necesidad de adecuar la intervención profesional a las particularidades específicas de cada persona, grupo o comunidad.

Los profesionales de la psicología recogen también en su código deontológico la no discriminación de personas por razón de nacimiento, edad, raza, sexo, credo, ideología, nacionalidad, clase social, o cualquier otra diferencia (art. 10). La American Psychological Association (2010) recoge, en el código de conducta y principios éticos de la profesión, el principio general de respeto de la dignidad y derechos de las personas, valorando la pertenencia cultural y las diferencias individuales.

Por último, el código deontológico de la profesión de Educación Social en España publicado por la Asociación Estatal de Educación Social (ASEDES, 
2004) contempla también como principio la igualdad, sin discriminación por razón de sexo, edad, religión, ideología, raza, idioma o cualquier otra diferencia (art. 8).

Desde el enfoque de la CI, el principio de igualdad debe completarse con la sensibilidad a las diferencias culturales, lo que requiere políticas que permitan disponer del encuadre y de los medios de actuación adecuados así como de modelos de intervención que contemplen planteamientos teóricos, estrategias, métodos y técnicas eficaces en este sentido.

Según Julve y Palomo (2005), los profesionales para ser culturalmente competentes deben tener formación en diversidad, ser conscientes del propio bagaje y perspectiva cultural y de la del otro, comprender la influencia que las características culturales juegan en los procesos de intervención y actuación profesional, evitar estereotipos y prejuicios, trabajar desde el respeto, el conocimiento, la aceptación de los propios límites, emplear técnicas y métodos que permitan el acercamiento y la comprensión mutua, y sobre todo, asentar y superar la sensibilidad intercultural (SI) para avanzar hacia la CI desde el compromiso por la igualdad.

Tomamos como punto de partida en este trabajo las dimensiones de la CI asumidas como relevantes en los profesionales de los servicios sociales comunitarios (Martínez et al., 2006) con una serie de supuestos de carácter práctico elaborados por el equipo de investigación y que se asocian con comportamientos concretos.

Los profesionales de los servicios sociales comunitarios andaluces, según el análisis realizado por el equipo de investigación en el marco del proyecto «Análisis de las necesidades formativas en los centros de servicios sociales de Andalucía» (del que también se extraen los datos de este estudio), tienen elevada CI global (en el 73 por ciento de los profesionales), sobre todo, en poseer habilidades de comunicación y relación intercultural adecuadas, tener conocimiento intercultural y desarrollar encuentros interculturales positivos (Vázquez, Fernández-Borrero y Álvarez, 2014:305).

Aunque estos profesionales tienen además alta Sensibilidad Intercultural (4,1 de media de cinco puntos), la misma no se traduce en interacciones culturalmente adaptadas,. Por esta razón, es necesario conocer la intervención intercultural en contextos de diversidad, abordando las competencias interculturales que se auto-atribuyen los trabajadores de los Servicios Sociales Comunitarios de Andalucía con las respuestas dadas a supuestos prácticos que actuarían como preguntas control. Las competencias, dimensiones y supuestos se explican y detallan en la descripción del instrumento. 
La construcción de estos casos o supuestos prácticos surge de la combinación de varios elementos: la formación y práctica profesional del Trabajo Social y la Psicología de miembros del equipo investigador, de la revisión bibliográfica realizada, y de la consulta y construcción conjunta y participativa con profesionales de los Servicios Sociales Comunitarios. Se trata por lo tanto de casos reales adaptados para el estudio, que pueden darse en cualquier centro de servicios sociales comunitarios ante los que los profesionales deben emitir un juicio sobre si la actuación profesional expuesta en cada uno de ellos es o no correcta.

Pretendemos dar respuesta a cuestiones como: ¿responder adecuadamente a los supuestos se asocia con disponer de elevados niveles de CI?, ¿qué dimensiones establecen asociaciones o cuáles de ellas adquieren más peso en otorgar mayor número de respuestas correctas a cada uno de supuestos presentados?, ¿existen diferencias debidas a características personales caracterización de los participantes que conlleven respuestas adecuadas a los supuestos? El objetivo de este trabajo es conocer las asociaciones entre las respuestas dadas a los supuestos prácticos con las características profesionales, el nivel de CI auto-atribuida y SI. Igualmente pretendemos conocer si se producen diferencias derivadas de pertenecer a una u otra profesión, tener distintos niveles de $\mathrm{CI}$ o características sociodemográficas diferentes (esto no está claro, ¿entre que se producen esas diferencias que se quieren constatar? en responder adecuadamente a los supuestos planteados. Como último objetivo planteamos la extracción de perfiles básicos en función de las variables objeto de estudio.

\section{Método}

\section{Participantes}

En este trabajo han participado 253 profesionales de los servicios sociales comunitarios en Andalucía de las diferentes profesiones que forman parte del equipo base: Trabajo Social, Psicología y Educación Social. Se ha realizado un muestreo de tipo estratificado con afijación proporcional según los puestos de trabajo, distribuyendo proporcionalmente el número total de trabajadores según los perfiles profesionales. 
100 Manuela-Ángela Fernández Borrero, Fernando Relinque-Medina y Susana Martí-García

Tabla 1. Caracterización de participantes

\begin{tabular}{|c|c|c|c|c|}
\hline & $\begin{array}{c}\text { Psicología } \\
(\mathrm{n}=38) \\
\%\end{array}$ & $\begin{array}{c}\text { Trabajo Social } \\
(\mathrm{n}=163) \\
\%\end{array}$ & $\begin{array}{c}\text { Educación Social } \\
(\mathrm{n}=52) \\
\%\end{array}$ & $\begin{array}{c}\text { TOTAL } \\
(\mathrm{N}=253) \\
\%\end{array}$ \\
\hline \multicolumn{5}{|l|}{ Género } \\
\hline Masculino & 19,35 & 7,89 & 24,24 & 15,0 \\
\hline Femenino & 80,65 & 92,11 & 75,76 & 85,0 \\
\hline \multicolumn{5}{|l|}{ Edad } \\
\hline $18-24$ & 0 & 1,23 & 0 & 0,7 \\
\hline $25-34$ & 21,05 & 30,25 & 11,54 & 23,3 \\
\hline $35-49$ & 65,79 & 64,20 & 82,69 & 69,3 \\
\hline $50-64$ & 13,16 & 4,32 & 5,77 & 6,8 \\
\hline \multicolumn{5}{|l|}{$\begin{array}{l}\text { Experiencia en } \\
\text { Servicios Sociales }\end{array}$} \\
\hline Menos de 5 años & 18,92 & 20,25 & 11,54 & 16,50 \\
\hline De 5 a 10 años & 43,24 & 30,67 & 25,00 & 28,96 \\
\hline De 11 a 20 años & 27,03 & 34,36 & 48,08 & 36,70 \\
\hline Más de 20 años & 10,81 & 14,72 & 15,38 & 17,85 \\
\hline \multicolumn{5}{|l|}{$\begin{array}{l}\text { Formación diversidad } \\
\text { cultural }\end{array}$} \\
\hline Sí & 39,47 & 60,63 & 50,00 & 55,48 \\
\hline No & 60,53 & 39,38 & 50,00 & 44,52 \\
\hline \multicolumn{5}{|l|}{ Idiomas } \\
\hline Sí & 76,32 & 61,96 & 75,00 & 67,45 \\
\hline No & 23,68 & 38,04 & 25,00 & 32,55 \\
\hline \multicolumn{5}{|l|}{ Relación } \\
\hline Sí & 81,58 & 69,33 & 84,62 & 73,49 \\
\hline No & 18,42 & 30,67 & 15,38 & 26,51 \\
\hline
\end{tabular}

\section{Instrumento}

El instrumento empleado en el estudio contiene diferentes escalas:

Preguntas sociodemográficas y de caracterización profesional: edad, sexo, experiencia profesional, formación en diversidad y contactos con personas de origen extranjero.

Una escala con nueve supuestos prácticos de actuación profesional que deben ser evaluados como correctos o incorrectos (con la opción también de no 
sabe/no contesta) por parte de los participantes, estando cada supuesto ligado a una o varias competencias interculturales. Estos supuestos pretenden descubrir el grado y/o nivel de competencias interculturales que los profesionales de la intervención ponen en juego en su praxis profesional. La correspondencia entre los supuestos y las competencias asociadas a la CI que reflejan así como la argumentación de la respuesta adecuada en cada uno se describen en el apéndice A.

\section{Apéndice A. SUPUESTOS PRÁCTICOS INCLUIDOS EN LA ENCUESTA EN RELACIÓN CON LAS COMPETENCIAS CULTURALES SEGÚN AUTORES}

\begin{tabular}{|c|c|c|c|}
\hline SUPUESTOS PRÁCTICOS & $\begin{array}{c}\text { COMPETENCIAS } \\
\text { CULTURALES } \\
\text { RELACIONADAS }\end{array}$ & AUTORES & $\begin{array}{l}\text { Argumentación de la respuesta } \\
\text { adecuada }\end{array}$ \\
\hline $\begin{array}{l}\text { 1. A su lugar de trabajo } \\
\text { llega un matrimonio. El } \\
\text { hombre se sienta enfrente } \\
\text { de Ud. y la mujer se queda } \\
\text { detrás. Lo primero que } \\
\text { harías sería pedirle a la } \\
\text { señora que se sentara al lado } \\
\text { de su esposo. }\end{array}$ & $\begin{array}{l}\text { «habilidad para no } \\
\text { enjuiciar, teniendo } \\
\text { conciencia de } \\
\text { las diferencias } \\
\text { culturales» }\end{array}$ & $\begin{array}{l}\text { Isidro Maya } \\
\text { (2002) } \\
\text { Cui Geng and } \\
\text { Njoku Awa } \\
\text { (1991) }\end{array}$ & $\begin{array}{l}\text { Incorrecto porque las personas } \\
\text { que mantengan relaciones } \\
\text { o trabajen en contextos } \\
\text { interculturales deben respetar } \\
\text { y aceptar estas diferencias } \\
\text { culturales. Es incorrecto que el/ } \\
\text { la profesional anime a la señora } \\
\text { a que se sitúe al mismo nivel que } \\
\text { su marido sin conocer y/o aceptar } \\
\text { las costumbres, valores, etc. } \\
\text { culturalmente diferentes. }\end{array}$ \\
\hline $\begin{array}{l}\text { 2. A su lugar de trabajo } \\
\text { acude una persona. Usted } \\
\text { tiene la mano derecha } \\
\text { ocupada en esos momentos } \\
\text { y le extiende la mano } \\
\text { izquierda a modo de saludo, } \\
\text { pero le rechaza. Esta } \\
\text { reacción le extraña. }\end{array}$ & $\begin{array}{l}\text { «comprender y } \\
\text { conocer nuestra } \\
\text { propia cultura» } \\
\text { «comprender y } \\
\text { conocer la/s otra/s } \\
\text { cultura/s» }\end{array}$ & $\begin{array}{l}\text { Maya (2002) } \\
\text { Richard } \\
\text { Wiseman, } \\
\text { Mitchell } \\
\text { Hammer and } \\
\text { Hiroko Nishida } \\
\text { (1989) }\end{array}$ & $\begin{array}{l}\text { Incorrecto, ya que según } \\
\text { los autores relacionados, es } \\
\text { incorrecto que a los profesionales } \\
\text { les extrañe este tipo de } \\
\text { reacciones, ya que se debe a que } \\
\text { no están formados ni poseen los } \\
\text { conocimientos necesarios acerca } \\
\text { de las culturas de los usuarios a } \\
\text { los que atienden. }\end{array}$ \\
\hline $\begin{array}{l}\text { 3. La persona que va a } \\
\text { verle no habla muy bien el } \\
\text { castellano. Para solventar } \\
\text { esta cuestión, usted llama a } \\
\text { un intérpretel mediador que } \\
\text { le ayude en la comunicación. }\end{array}$ & $\begin{array}{l}\text { «tolerar la } \\
\text { ambigüedad y la } \\
\text { incertidumbre» }\end{array}$ & $\begin{array}{l}\text { Geng and Awa } \\
\text { (1991) } \\
\text { Maya (2002) }\end{array}$ & $\begin{array}{l}\text { Incorrecto porque en situaciones } \\
\text { poco definidas e inciertas donde } \\
\text { la persona no domina muy } \\
\text { bien el idioma, el nerviosismo } \\
\text { del profesional hace que } \\
\text { recurra a otros profesionales } \\
\text { como intérpretes, traductores, } \\
\text { Siguiendo a los autores citados, } \\
\text { esto genera indefensión y } \\
\text { desconfianza en el usuario y } \\
\text { la calidad de la intervención } \\
\text { disminuye. }\end{array}$ \\
\hline $\begin{array}{l}\text { 4. Cada vez que usted } \\
\text { habla sobre métodos de } \\
\text { planificación familiar, } \\
\text { la señora china que está } \\
\text { enfrente suya, sonrie. Usted } \\
\text { cree que le está entendiendo } \\
\text { y le explica con detalle } \\
\text { algunos de estos métodos. }\end{array}$ & $\begin{array}{l}\text { «flexibilidad } \\
\text { cognitiva» } \\
\text { «empatía cultural» } \\
\text { «comprender y } \\
\text { conocer la/s otra/s } \\
\text { cultura/s» }\end{array}$ & $\begin{array}{l}\text { Maya (2002) } \\
\text { Geng and Awa } \\
\text { (1992) }\end{array}$ & $\begin{array}{l}\text { Es incorrecto que el profesional } \\
\text { continúe explicando } \\
\text { detalladamente los métodos } \\
\text { anticonceptivos debido a que } \\
\text { no está suspendiendo su juicio } \\
\text { momentáneamente, ni situándose } \\
\text { con más facilidad en la postura } \\
\text { de la usuaria, ni comprendido la } \\
\text { cultura de la señora. }\end{array}$ \\
\hline
\end{tabular}

Alternativas. Cuadernos de Trabajo Social, 24, 2017, pp. 91-118 - ISSN 1133-0473 
5. La hija de un matrimonio recién divorciado cree que su madre no debería haber dado el paso de solicitar el divorcio. Tú le explicas que eso es normal aqui y que hay muchos niños con los padres divorciados, y no pasa nada

6. En una visita a domicilio, ante sus preguntas sobre la situación familiar, la señora le pregunta a usted si está casada-o, si tiene hijos... Usted no le da esta información porque es una cuestión personal que no afecta a la intervención.

\section{Cuando le comenta} al señor que puede tener derecho a determinados recursos, él le dice siempre que lo que Dios quiera, que lo que Dios quiera... Usted le dice que no, que es algo a lo que tiene derecho.

8. La persona con la que habla está muy cerca de usted hasta el punto que intenta siempre poner la mano sobre su brazo. Usted lo retira porque se siente incómodo/a.

9. Realiza una visita a
domicilio. El señor le atiende
en el salón y la señora se
queda en la cocina. Usted,
que quiere hablar con los
dos, alza la voz para que la
señora le oiga y le responda.

«habilidad para formar y mantener relaciones» «mantener una distancia social apropiada»

«habilidad para comprender y conocer nuestra propia cultura» «habilidad para comprender y conocer las demás culturas» «flexibilidad cognitiva»

«mantener una distancia social apropiada»

«habilidad para la resolución de conflictos»
Maya (2002)

Geng and Awa (1992)

Maya (2002)

Wiseman et al (1989)

Geng and Awa (1992)

Wiseman et al (1989)

Maya (2002)

Geng and Awa (1992) Maya (2002)
Incorrecto porque no está empatizando culturalmente con la protagonista de la intervención y por consiguiente, no está poniéndose en su lugar, respetándola y aceptando su manera de ver y entender el mundo.

Es incorrecto que se responda a la usuaria con que es una cuestión personal debido a que estas competencias son desarrolladas por diversos autores mencionados como un elemento de vital importancia para identificar y gestionar las necesidades personales y el apoyo, y establecer contacto y mantener relaciones con personas extranjeras.

Adquirir conocimientos culturales (generales y específicos) ayuda a interactuar de forma positiva y eficaz con personas de diferentes culturas. Es incorrecta la actitud seguida por el/la profesional, no dejando que la persona elija libremente si desea o no los recursos a los que tiene derecho, aunque el rechazo sea por cuestiones únicamente culturales.

Incorrecto porque cada cultura da una relevancia diferente a la distancia que debe existir entre profesional y usuario. Es importante entender cuál es la distancia adecuada que puede determinar la conformación y mantenimiento de las relaciones sociales con los demás.

Incorrecto porque es necesario re conocer de manera adecuada al interlocutor si queremos tener éxito en la interacción comunicativa. Implicar a otra persona que no está próxima no solo dificulta la comunicación, sino que niega la interlocución de quien tenemos delante.

Elaboración propia

Alternativas. Cuadernos de Trabajo Social, 24, 2017, pp. 91-118 - ISSN 1133-0473 
Una escala de cinco ítems tipo Likert para valorar las dimensiones de la CI auto-atribuida, descritas por Martínez et al. (2006): grado de conciencia intercultural, deseo por el encuentro intercultural, grado de conocimiento cultural, desarrollo de habilidades culturales y encuentro intercultural. Las preguntas son valoradas a partir de una graduación de cuatro posibilidades (Purnell y Paulanka, 1998), donde el uno responde a la incompetencia inconsciente y el sujeto muestra ceguera cultural al no tener constancia de su falta de conocimientos, conciencia, etc.; el dos al de incompetencia consciente que implica que la persona percibe su hándicap cultural pero sus conocimientos al respecto son escasos; tres a competencia consciente que indica que el sujeto hace un esfuerzo voluntario por hacer suyos ciertos conocimientos y pautas culturales con respecto a su cultura y a las otras y es capaz de ofrecer respuestas profesionales culturalmente sensibles; y cuatro a competencia inconsciente como habilidad interiorizada (prácticamente automatizada) para prestar servicios y ofrecer respuestas culturalmente sensibles según las características de cada sujeto.

Otra escala tipo Likert de grado de acuerdo de cinco puntos sobre aspectos de intervención de los profesionales con personas de origen extranjero, tomada de Martínez et al. (2006), que incluye: ser consciente de los propios sesgos y prejuicios hacia grupos culturales diferentes; poseer habilidades para gestionar los aspectos de diversidad en las situaciones profesionales; tener conocimientos para percibir los aspectos de diversidad en estas situaciones profesionales; tener los conocimientos necesarios para diseñar un proyecto de intervención teniendo en cuenta los elementos de diversidad cultural; y tener conocimientos suficientes sobre los elementos de diversidad de las personas usuarias.

Se incluye también la traducción en castellano de la Escala de SI Vilá, 2005) propuesta por Chen y Starosta (2000), de 24 ítems y cinco dimensiones: implicación, respeto, confianza, disfrute y atención.

\section{Estrategia de análisis}

Realizamos una caracterización básica inicial de los participantes en las diferentes escalas: $\mathrm{CI}$ auto-atribuida, competencias específicas y aspectos relacionados con la intervención culturalmente adecuada.

Los análisis principales se basan en los supuestos prácticos y en las competencias relacionadas, habiéndose realizado correlaciones ( $\chi^{2}$ Pearson), asociaciones, comparaciones de medias y análisis de la varianza. Se emplea la $d$ de Cohen (Cohen 1988) para el cálculo de los tamaños de efectos, considerando 
los valores de $d$ hasta 0.20 como efectos pequeños, hasta 0.50 como tamaños del efecto medianos y hasta 0.80 o más como elevados.

Finalmente se realiza un Análisis Factorial de Correspondencias Múltiples (AFC). Se trata de un análisis multivariante de carácter exploratorio, para la extracción de clases, que permite elaborar grupos de perfiles de los participantes según las variables claves del estudio.

Para el análisis y tratamiento de los datos empleamos el software estadístico SPSS 20.0. También utilizamos el software Anaconda pertenecientes al grupo de herramientas Catalyse, que permite hacer un análisis cuantitativo de los datos, combinando el análisis factorial de correspondencias y la clasificación jerárquica ascendente. Este grupo de herramientas Catalyse surgen del trabajo de la Red Europea de Inteligencia Territorial ${ }^{1}$ y se emplean para el desarrollo de diagnósticos y evaluación de datos individuales para definir perfiles o tipologías, que permitan ofrecer visiones conjuntas de datos y variables.

\section{Resultados}

Inicialmente se muestran los resultados descriptivos básicos para los supuestos y los análisis de varianzas y asociaciones entre estos supuestos y las características sociodemográficas. Presentamos igualmente las asociaciones, correlaciones y diferencias significativas en relación con los supuestos de las variables claves del estudio como la CI, competencias de intervención intercultural y SI.

\section{Análisis de los supuestos estudiados}

El índice de acierto de los supuestos ha sido variable, oscilando entre el 15, $8 \%$ (del supuesto tres) y el 94,3\% (del supuesto cuatro), con un nivel medio de respuestas correctas de 4 supuestos del total de 9 presentados.

Un 42,1 de los participantes se sitúan en un nivel bajo de respuestas correctas (tres o menos), un 48,9 por ciento en nivel medio (de cuatro a seis respuestas adecuadas) y el resto, un 9,1 por ciento, en nivel alto (siete o más supuestos adecuadamente respondidos).

1. European Network of Territorial Intelligence (ENTI) integra la investigación europea actual sobre las herramientas de inteligencia territorial. 
Tabla 2. Respuestas dadas a los supuestos según profesiones

\begin{tabular}{lcccc}
\hline & $\begin{array}{c}\text { Psicología } \\
(\mathrm{n}=38) \\
\%\end{array}$ & $\begin{array}{c}\text { Trabajo } \\
\text { Social } \\
(\mathrm{n}=163) \\
\%\end{array}$ & $\begin{array}{c}\text { Educación } \\
\text { Social } \\
(\mathrm{n}=52) \\
\%\end{array}$ & $\begin{array}{c}\text { TOTAL } \\
(\mathrm{N}=253) \\
\%\end{array}$ \\
\hline Respuesta adecuada (\%) & & & & \\
Supuesto 1 matrimonio & 43,8 & 24,3 & 28,3 & 26,5 \\
Supuesto 2 manos & 70,4 & 59,4 & 81,1 & 66,0 \\
Supuesto 3 mediación & 9,4 & 14,0 & 25,0 & 15,8 \\
Supuesto 4 china & 90,0 & 78,3 & 83,3 & 82,9 \\
Supuesto 5 divorcio & 53,1 & 35,2 & 31,8 & 35,8 \\
Supuesto 6 personal & 50,0 & 50,0 & 68,9 & 56,9 \\
Supuesto 7 derecho & 41,4 & 33,6 & 33,3 & 34,3 \\
Supuesto 8 cercanía & 74,1 & 52,3 & 57,1 & 59,6 \\
Supuesto 9 gritar & 93,8 & 94,2 & 92,2 & 94,3 \\
Niveles de respuestas adecuadas & & & & \\
Bajo (0-3) & 31,6 & 48,8 & 38,5 & 42,1 \\
Medio (4-6) & 60,5 & 43,2 & 48,1 & 48,8 \\
Alto (7 a 9) & 7,9 & 8 & 13,5 & 9,1 \\
\hline
\end{tabular}

La profesión no produce diferencias significativas en el número medio de supuestos respondidos correctamente, aunque los trabajadores sociales tienen 3,8 respuestas correctas de media, frente a 4,2 de los educadores sociales y psicólogos. La variable edad determina una asociación significativa entre las personas de mayor edad (de 50 a 64 años) con responder inadecuadamente al cuarto supuesto planteado, sobre la explicación de métodos de planificación familiar ante una persona extranjera china que sólo sonríe $(\chi 2(3, N=191)=$ $8,57, \mathrm{p}=0,036)$ y al quinto sobre normalización del divorcio $(\chi 2(3, \mathrm{~N}=217)=$ $8,06, \mathrm{p}=0,045)$, que reflejan la competencia de comprender y conocer otras culturas y la empatía cultural.

Tener formación en diversidad cultural se asocia significativamente con responder adecuadamente el sexto supuesto que versa sobre la preservación de la información personal del profesional ante la persona usuaria $(\chi 2(3, \mathrm{~N}=$ $194)=3,99, p=0,046)$ y que refleja la habilidad de relación y el manejo adecuado de la distancia social. Además, las personas que mantienen algún tipo de relación con personas extranjeras se asocian con la respuesta adecuada de este mismo supuesto $(\chi 2(1, \mathrm{~N}=213)=4,43, \mathrm{p}=0,026)$ y del supuesto séptimo donde el profesional explica los derechos de las personas usuarias $(\chi 2(1, \mathrm{~N}=$ $211)=3,48, p=0,043)$ mostrando la habilidad de conocer y comprender tanto la cultura propia como ajenas, así como la flexibilidad cognitiva. 


\section{Resultados sobre competencia intercultural}

En líneas generales La CI global es elevada, sobre todo a nivel consciente y destacando un nivel más alto en los psicólogos. En las dimensiones de conciencia y de motivación por el encuentro destaca la máxima competencia (inconsciente) de los educadores sociales frente a otros profesionales. En el resto de dimensiones (conocimiento, desarrollo de habilidades y encuentro) las tres profesiones muestran niveles similares. No se producen diferencias estadísticamente significativas entre las profesiones.

Tabla 3. Dimensiones (graduación) de la Competencia intercultural autoatribuida por profesiones

\begin{tabular}{|c|c|c|c|c|c|c|c|}
\hline & & $\begin{array}{c}\text { Conciencia } \\
\text { (\%) }\end{array}$ & $\begin{array}{l}\text { Motivación por } \\
\text { el encuentro } \\
\text { intercultural(\%) }\end{array}$ & $\begin{array}{c}\text { Conocimiento } \\
(\%)\end{array}$ & $\begin{array}{c}\text { Desarrollo } \\
\text { habilidades } \\
\text { (\%) }\end{array}$ & $\begin{array}{c}\text { Encuentro } \\
\text { intercultural } \\
\text { (\%) }\end{array}$ & $\begin{array}{c}\text { Competencia } \\
\text { global (\%) }\end{array}$ \\
\hline \multirow{4}{*}{$\begin{array}{c}\text { Total } \\
(\mathrm{N}=253)\end{array}$} & $\begin{array}{l}\text { Incompetencia } \\
\text { inconsciente }\end{array}$ & 29,2 & 34,2 & 1,0 & 4,1 & 6,1 & 0,7 \\
\hline & $\begin{array}{l}\text { Incompetencia } \\
\text { consciente }\end{array}$ & 14,2 & 12,4 & 27,3 & 14,9 & 28,4 & 10,7 \\
\hline & $\begin{array}{l}\text { Competencia } \\
\text { consciente }\end{array}$ & 49,5 & 15,1 & 66,0 & 62,4 & 38,5 & 64,6 \\
\hline & $\begin{array}{l}\text { Competencia } \\
\text { inconsciente }\end{array}$ & 7,1 & 38,3 & 5,7 & 18,6 & 27,0 & 24,1 \\
\hline \multirow{4}{*}{$\begin{array}{l}\text { Psicólogos/as } \\
\qquad(\mathrm{N}=38)\end{array}$} & $\begin{array}{l}\text { Incompetencia } \\
\text { inconsciente }\end{array}$ & 24,3 & 47,4 & 0 & 2,7 & 0 & 0 \\
\hline & $\begin{array}{l}\text { Incompetencia } \\
\text { consciente }\end{array}$ & 13,5 & 15,8 & 28,9 & 13,5 & 27,0 & 8,3 \\
\hline & $\begin{array}{l}\text { Competencia } \\
\text { consciente }\end{array}$ & 56,8 & 13,2 & 63,2 & 67,6 & 43,2 & 75,0 \\
\hline & $\begin{array}{l}\text { Competencia } \\
\text { inconsciente }\end{array}$ & 5,4 & 23,7 & 7,9 & 16,2 & 29,7 & 16,7 \\
\hline \multirow{4}{*}{$\begin{array}{c}\text { Trabajadores } \\
\text { sociales } \\
(\mathrm{N}=163)\end{array}$} & $\begin{array}{l}\text { Incompetencia } \\
\text { inconsciente }\end{array}$ & 32,3 & 31,3 & 1,8 & 4,3 & 6,8 & 1,3 \\
\hline & $\begin{array}{l}\text { Incompetencia } \\
\text { consciente }\end{array}$ & 16,1 & 11,7 & 27,0 & 16,8 & 31,5 & 11,9 \\
\hline & $\begin{array}{l}\text { Competencia } \\
\text { consciente }\end{array}$ & 43,5 & 17,2 & 65,6 & 59,0 & 35,2 & 62,3 \\
\hline & $\begin{array}{l}\text { Competencia } \\
\text { inconsciente }\end{array}$ & 8,1 & 39,9 & 5,5 & 19,9 & 26,5 & 24,5 \\
\hline \multirow{4}{*}{$\begin{array}{l}\text { Educadores } \\
\text { sociales } \\
(\mathrm{N}=52)\end{array}$} & $\begin{array}{l}\text { Incompetencia } \\
\text { inconsciente }\end{array}$ & 23,1 & 32,7 & 0 & 3,8 & 1,9 & 0 \\
\hline & $\begin{array}{l}\text { Incompetencia } \\
\text { consciente }\end{array}$ & 9,6 & 13,5 & 35,3 & 15,4 & 23,1 & 9,8 \\
\hline & $\begin{array}{l}\text { Competencia } \\
\text { consciente }\end{array}$ & 61,5 & 13,5 & 62,7 & 65,4 & 51,9 & 68,6 \\
\hline & $\begin{array}{l}\text { Competencia } \\
\text { inconsciente }\end{array}$ & 5,8 & 40,4 & 2,0 & 15,4 & 23,1 & 21,6 \\
\hline
\end{tabular}


Los análisis han arrojado algunas asociaciones significativas entre dimensiones de la CI con determinados supuestos. De este modo, responder adecuadamente al segundo supuesto se asocia con el mayor nivel de competencia de la conciencia intercultural $(\chi 2(3, N=194)=8,90, p=0,031)$. Además, responder adecuadamente a un número elevado de supuestos ( 7 o más) se asocia con la competencia consciente de la conciencia intercultural ( $\chi 2(6, \mathrm{~N}$ $=249)=23,29, \mathrm{p}=0,001$ ), y un nivel bajo de respuestas con la incompetencia. Los tamaños de efecto son pequeños a pesar de la significatividad estadística ( $V$ de Cramer de 0,21 y de 0,29 respectivamente).

El nivel de competencia consciente de la motivación por el encuentro intercultural se asocia significativamente con responder adecuadamente al primer supuesto, que sería calificar como incorrecto que el profesional invite a la mujer usuaria a sentarse al lado de su marido $(\chi 2(3, N=230)=9,13, p=0,018 ; \mathrm{V}$ de Cramer $=0,20)$ reflejando la habilidad de no enjuiciar. Esta elevada motivación también se asocia con el nivel alto de supuestos respondidos adecuadamente $(\chi 2(6, \mathrm{~N}=252)=15,10 \mathrm{p}=0,019 ; \mathrm{V}$ de Cramer=0,17).

El nivel máximo de competencia en conocimiento cultural se asocia de manera significativa con la respuesta adecuada al segundo supuesto, que versaba sobre un rechazo o reparo de la persona usuaria ante un modo de saludo del profesional $(\chi 2(3, \mathrm{~N}=196)=9,48 \mathrm{p}=0,024 ; \mathrm{V}$ de Cramer $=0,220)$ y que refleja la habilidad de comprender y conocer tanto la cultura propia como otras diferentes.

Por otra parte, ser incompetentes para el encuentro intercultural se asocia con responder inadecuadamente al supuesto octavo, que recoge un ejemplo de incomodidad del profesional ante una escasa distancia social de la persona usuaria $(\chi 2(3, N=197)=9,09 p=0,028 ; \mathrm{V}$ de Cramer $=0,215)$.

La dimensión de desarrollo de habilidades no se asocia significativamente con ningún supuesto.

Por último, la $\mathrm{CI}$, considerada a nivel global, se asocia con el segundo supuesto (rechazo ante el saludo con la mano izquierda del profesional) $(\chi 2$ $(3, \mathrm{~N}=192)=8,56 \mathrm{p}=0,036 ; \mathrm{V}$ de Cramer $=0,211)$, de modo que la diferencia entre responder adecuadamente o no se produce en los niveles máximos de competencia, siendo los de mayor nivel (competencia inconsciente) los que dan respuestas correctas y los que tienen un nivel algo menor, aunque elevado (competencia consciente) los que responden erróneamente. También se produce asociación con el octavo supuesto que exponía el rechazo profesional a una escasa distancia social de la persona usuaria, estableciéndose la asociación 
el nivel de incompetencia intercultural y responder inadecuadamente ( $\chi 2$ (3, $\mathrm{N}=192)=8,65 \mathrm{p}=0,034 ; \mathrm{V}$ de Cramer=0,212).

En cuestiones relativas a la intervención destaca positivamente las habilidades en la gestión de la diversidad, mientras que la menor media se presenta en la conciencia de los propios sesgos y prejuicios. Los trabajadores sociales muestran, frente a educadores sociales, mayor percepción de los aspectos de diversidad presentes en situaciones profesionales $(F(2,244)=3,43 p=0,034$, $d=.69$, IC $95 \%[.02, .77])$.

Los profesionales que responden adecuadamente al primer supuesto sobre habilidad de no enjuiciar (el profesional le pide a la mujer de un matrimonio heterosexual que se siente al lado de su marido), son significativamente más conscientes de sus propios sesgos o prejuicios $(t(218)=2,28, p=0,023, d=.58$, IC $95 \%[0,067,0,918])$ que aquellos que responden incorrectamente.

Los que responden adecuadamente al supuesto octavo, sobre la distancia social, tienen significativamente mejor puntuación en la escala global de intervención $(t(193)=2,15, p=0,033, d=.31$, IC 95\% [0,018, 0,409]) y mayor media en habilidades de gestión de la diversidad en situaciones profesionales $(t(191)=2,83, p=0,005, d=.40$, IC $95 \%[0,108,0,604])$ que aquellos que responden de manera inadecuada.

Por otra parte, la SI es bastante elevada $(M=4,1 ; S D=0,44)$, destacando un elevado respeto por las diferencias culturales (tabla 3). El total de respuestas correctas a los supuestos no correlaciona con las puntuaciones en SI global ni en cada una de sus dimensiones. Únicamente se han encontrado diferencias estadísticamente significativas en la implicación en relación con la respuesta al segundo supuesto, siendo mayor la implicación para los que responden adecuadamente $(t(141)=0,18, p=0,011, d=.46$, IC 95\% $[0,058,0,438])$. 
Tabla 4. Aspectos relacionados con competencia intercultural y Sensibilidad Intercultural

\begin{tabular}{|c|c|c|c|c|c|c|c|c|}
\hline \multirow[b]{2}{*}{ INTERVENCIÓN } & \multicolumn{2}{|c|}{$\begin{array}{c}\text { Total } \\
(\mathrm{N}=253)\end{array}$} & \multicolumn{2}{|c|}{$\begin{array}{l}\text { Psicólogos/as } \\
\quad(\mathrm{N}=38)\end{array}$} & \multicolumn{2}{|c|}{$\begin{array}{c}\text { Trabajador/a } \\
\text { social } \\
(N=163)\end{array}$} & \multicolumn{2}{|c|}{$\begin{array}{c}\text { Educadores/as } \\
\text { sociales } \\
(\mathrm{N}=52)\end{array}$} \\
\hline & Mean & SD & Mean & SD & Mean & SD & Mean & SD \\
\hline Conciencia de prejuicios & 3,0 & 1,46 & 3,29 & 1,384 & 2,91 & 1,490 & 3,23 & 1,403 \\
\hline Habilidades gestión diversidad & 3,7 &, 90 & 3,78 & ,929 & 3,76 & ,895 & 3,52 & ,863 \\
\hline Conocimiento percibir diversidad & 3,4 &, 95 & 3,44 & ,998 & 3,50 & ,927 & 3,10 & ,931 \\
\hline $\begin{array}{l}\text { Conocimiento para diseño de proyectos } \\
\text { intervención }\end{array}$ & 3,1 & 1,10 & 3,03 & 1,158 & 3,15 & 1,115 & 2,86 & ,990 \\
\hline Conocimiento sobre las diferencias de usuarios & 3,3 & 1,02 & 3,36 & 1,175 & 3,29 & ,996 & 3,18 & 1,004 \\
\hline Competencia Intervención Intercultural (Suma) & 3,3 & ,68 & 3,33 &, 780 & 3,32 & ,668 & 3,18 & ,639 \\
\hline \multicolumn{9}{|l|}{ SENSIBILIDAD INTERCULTURAL } \\
\hline Implicación & 4,1 &, 55 & 4,1 &, 51 & 4,1 &, 59 & 4,2 & 47 \\
\hline Respeto & 4,5 &, 54 & 4,5 & 48 & 4,5 &, 58 & 4,6 &, 41 \\
\hline Confianza & 3,7 & ,63 & 3,8 & ,69 & 3,7 & ,62 & 3,5 &, 60 \\
\hline Disfrute & 4,1 &, 88 & 4,4 &, 57 & 4,0 &, 90 & 4,1 & ,93 \\
\hline Atención & 4,0 & ,69 & 4,1 &, 75 & 3,9 &, 70 & 4,1 &, 55 \\
\hline Global & 4,1 &, 44 & 4,2 &, 33 & 4,1 &, 48 & 4,1 &, 36 \\
\hline
\end{tabular}

Análisis tipológico de los resultados

Posteriormente efectuamos un Análisis Factorial de Correspondencias, para realizar un análisis tipológico de los participantes en función de su caracterización, sus puntuaciones en las variables claves y en los supuestos.

En este análisis cada uno de los ejes está determinado fundamentalmente por una variable o conjunto de ellas. El eje 1, distribuye según los niveles de incompetencia y competencia de las dimensiones de CI, aunque también lleva asociado los niveles de SI. El eje 2 se relaciona de un modo más directo con las respuestas adecuadas a los supuestos prácticos planteados y, el eje 3, distribuye según posesión o no de los aspectos de intervención profesional. Los resultados muestran un total de cuatro clases diferenciadas, donde la primera y segunda clase son las más positivas.

La clase uno concentra la mayoría de psicólogos participantes y viene definida fundamentalmente por el eje tres. Son individuos con conocimiento de otros idiomas y con contacto cotidiano con personas extranjeras. Están de acuerdo en tener habilidades en intervención y en percibir la diversidad cultural de las personas y situaciones en las que trabajan. Saben realizar proyectos en estos contextos y disponen de conocimientos sobre la praxis intercultural. Además, esta clase aúna las respuestas adecuadas a la mayoría de los supuestos. 
La clase dos, también positiva, viene marcada fundamentalmente por el eje 1. Se caracteriza por el mayor nivel de competencia en todas las dimensiones de CI (menos la motivación). A esto se añade la respuesta adecuada a tres supuestos (segundo, cuarto y noveno), lo que le acerca a la clase uno, así como la presencia de los niveles elevados de SI, fundamentalmente de implicación, respeto y atención.

La clase tres, más asociada al eje tres, se caracteriza por la neutralidad en los aspectos de intervención en contextos de interculturalidad. Tienen formación en diversidad pero con nivel de incompetencia en la dimensión de conciencia intercultural, pero competencia en la dimensión de motivación de la CI. El nivel de respuestas adecuadas en los supuestos es bajo (4 supuestos de 9).

La clase cuatro es la que muestra los niveles de incompetencia de la CI, así como los niveles medios de SI. Se encuentran alejados de las respuestas correctas a los supuestos. Además, no tienen contacto con personas extranjeras y no tienen conocimientos de otros idiomas.

Siendo el grueso de edad de la muestra de 35 a 49 años, esta franja se localiza en mayor proporción en la clase tercera. En cuanto al sexo, en todas las clases destacan las mujeres dado que el 87 por ciento del total de la muestra son mujeres, mientras que el porcentaje de hombres se aglutina en las dos primeras clases (las más positivas).

Tabla 5. Características de identificación por clases (distribución por filas y columnas)

\begin{tabular}{|c|c|c|c|c|c|c|c|c|}
\hline & \multicolumn{4}{|c|}{$\%$ columnas } & \multicolumn{4}{|c|}{$\%$ filas } \\
\hline & $\begin{array}{l}\text { Clase } 1 \\
(\mathrm{~N}=52)\end{array}$ & $\begin{array}{c}\text { Clase } \\
2 \\
(\mathrm{~N}=73)\end{array}$ & $\begin{array}{l}\text { Clase } 3 \\
(\mathrm{~N}=81)\end{array}$ & $\begin{array}{c}\text { Clase } 4 \\
(\mathrm{~N}=47\end{array}$ & Clase 1 & Clase 2 & Clase 3 & Clase 4 \\
\hline Clase & & & & & 20,6 & 28,9 & 32 & 18,6 \\
\hline \multicolumn{9}{|l|}{ Género } \\
\hline Masculino & 18,2 & 18,0 & 2,1 & 13,9 & 34,8 & 39,1 & 4,3 & 21,7 \\
\hline Femenino & 81,8 & 82,0 & 97,9 & 86,1 & 23,2 & 26,5 & 30,3 & 20,0 \\
\hline \multicolumn{9}{|l|}{ Edad } \\
\hline $18-24$ & 0 & 1,4 & 0 & 2,1 & 0 & 50,0 & 0 & 50,0 \\
\hline $25-34$ & 31,4 & 27,4 & 24,7 & 14,9 & 25,4 & 31,7 & 31,7 & 11,1 \\
\hline $35-49$ & 56,9 & 69,9 & 67,9 & 78,7 & 16,9 & 29,7 & 32,0 & 21,5 \\
\hline $50-64$ & 11,8 & 1,4 & 7,4 & 4,3 & 40,0 & 6,7 & 40,0 & 13,3 \\
\hline \multicolumn{9}{|c|}{ Formación en diversidad } \\
\hline Sí & 61,5 & 65,3 & 55,1 & 32,6 & 23,4 & 34,3 & 31,4 & 10,9 \\
\hline No & 38,5 & 34,7 & 44,9 & 67,4 & 18,0 & 22,5 & 31,5 & 27,9 \\
\hline \multicolumn{9}{|l|}{ Relación } \\
\hline Sí & 94,2 & 82,2 & 63,0 & 59,6 & 26,1 & 31,9 & 27,1 & 14,9 \\
\hline No & 5,8 & 17,8 & 37,0 & 40,4 & 4,6 & 20,0 & 46,2 & 29,2 \\
\hline \multicolumn{9}{|l|}{ Idioma } \\
\hline Sí & 88,5 & 69,9 & 60,5 & 48,9 & 27,2 & 30,2 & 29,0 & 13,6 \\
\hline No & 11,5 & 30,1 & 39,5 & 51,1 & 7,1 & 26,2 & 38,1 & 28,6 \\
\hline
\end{tabular}


Figura 1. Distribución espacial de clases en los diferentes planos/ejes

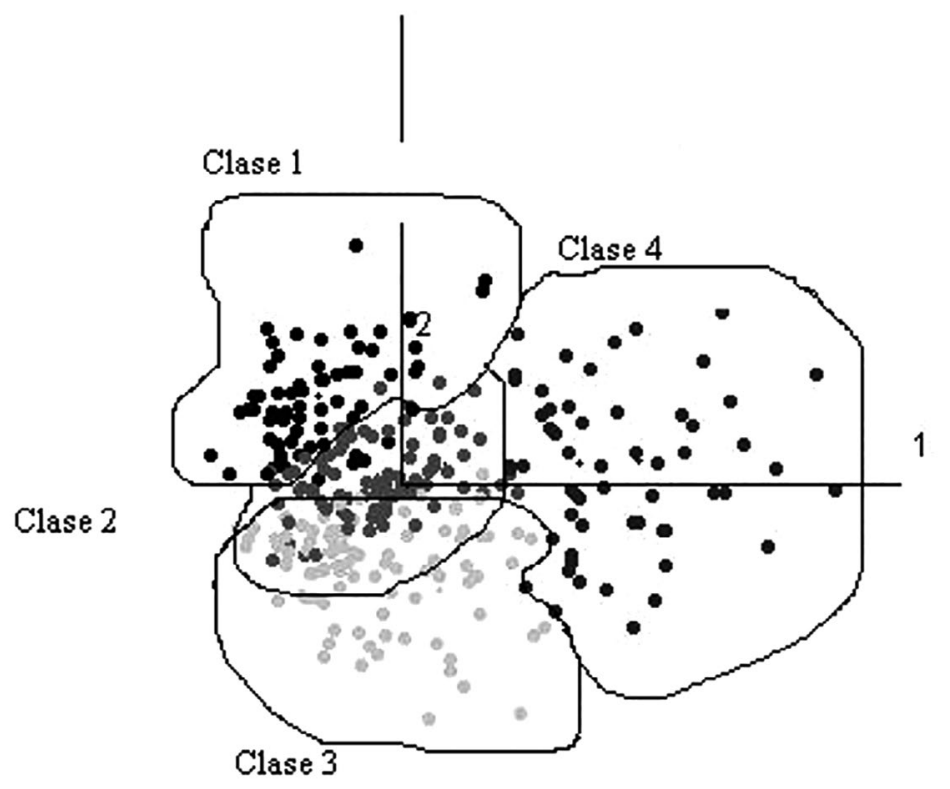

El número medio de supuestos respondidos adecuadamente es mayor en las primeras clases, teniendo la clase uno la media más elevada con 5 supuestos y la tercera clase la más baja con 2,5. Existen diferencias significativas en estas medias entre las clases $(F(3,251)=39,84 p \leq .001)$, de modo que la clase tres y cuatro se diferencian de todas las restantes, estableciéndose tres subconjuntos, uno formado por la clase uno y dos, otro formado por la clase tres y otro por la cuatro. Además, la primera clase se asocia significativamente con el nivel elevado de respuestas correctas (7 o más supuestos adecuados), la segunda clase con el nivel medio y la tercera con el nivel bajo. La cuarta clase tiene su mayor porcentaje en el nivel bajo, aunque no se asocia estadísticamente.

Responder adecuadamente a los supuestos se asocia significativamente con pertenecer a la primera clase, a excepción del segundo, cuarto y quinto donde las respuestas adecuadas se asocian con pertenecer a la segunda clase. La clase tercera se asocia con las respuestas inadecuadas en todos los supuestos a excepción del segundo. Cabe mencionar que el supuesto nueve no muestra asociación estadísticamente significativa con ninguna clase. 
Posteriormente se han analizado las clases en relación con las variables claves como CI, SI y aspectos de intervención. Las medias más elevadas de los aspectos de intervención se dan en la clase primera, a excepción de ser consciente de los propios sesgos que se ubica en la segunda clase. Las medias más bajas en su mayoría se presentan en la cuarta clase.

En relación con la CI, la mayor media se sitúa en la clase dos (considerando desde 1 nivel de incompetencia a 4 nivel competencia) y la menor en la clase cuatro. Se producen asociaciones entre los niveles de competencia de las dimensiones de conocimiento y habilidades con la primera clase y de conciencia, motivación, encuentro y del total de CI con la segunda. Los niveles de incompetencia de todas las dimensiones y del total de CI se asocian con la cuarta clase.

Si tomamos la SI tanto de manera conjunta como en cada una de sus dimensiones, las mayores medias se ubican en la primera clase y las menores (que se sitúan en nivel medio de la escala) en la cuarta clase. Se establecen asociaciones significativas entre los niveles medios de todas ellas y del total de la escala con la cuarta clase y de los niveles altos de confianza, disfrute y del total de SI con la primera y de la dimensión de implicación con la segunda clase.

Tabla 6. Asociaciones entre clases y respuestas a los supuestos prácticos

\begin{tabular}{|c|c|c|c|c|}
\hline & $\begin{array}{c}\text { Asociación } \\
\text { con responder } \\
\text { adecuadamente }\end{array}$ & $\begin{array}{c}\text { Asociación } \\
\text { con responder } \\
\text { inadecuadamente }\end{array}$ & Significancia estadística & $\begin{array}{l}\text { Coeficiente de } \\
\text { contingencia }\end{array}$ \\
\hline Supuesto 1 & Clase 1 & Clase 3 & $\chi 2(3, N=230)=17,54 \mathrm{p}=.001$ & 0,266 \\
\hline Supuesto 2 & Clase 2 & Clase 4 & $\chi 2(3, \mathrm{~N}=197)=22,21 \mathrm{p} \leq .001$ & 0,318 \\
\hline Supuesto 3 & Clase 1 & Clase 3 & $\chi^{2}(3, \mathrm{~N}=230)=16,70 \mathrm{p}=.001$ & 0,260 \\
\hline Supuesto 4 & Clase 2 & & $\chi 2(3, N=192)=15,07 \mathrm{p}=.002$ & 0,270 \\
\hline Supuesto 5 & & & $\chi 2(3, \mathrm{~N}=218)=30,52 \mathrm{p} \leq .001$ & 0,350 \\
\hline Supuesto 6 & Clase 1 & & $\chi 2(3, N=213)=20,05 \mathrm{p} \leq .001$ & 0,293 \\
\hline Supuesto 7 & & & $\chi 2(3, \mathrm{~N}=211)=24,55 \mathrm{p} \leq .001$ & 0,323 \\
\hline Supuesto 8 & & Clase 3 y Clase 4 & $\chi 2(3, N=197)=24,60 \mathrm{p} \leq .001$ & 0,333 \\
\hline Supuesto 9 & \multirow{2}{*}{\multicolumn{2}{|c|}{ No asoc. }} & No asociación significativa & \\
\hline $\begin{array}{c}\text { TOTAL } \\
\text { SUPUESTOS }\end{array}$ & & & $\chi 2(6, N=253)=118,07 \mathrm{p} \leq .001$ & 0,565 \\
\hline
\end{tabular}




\begin{tabular}{|c|c|c|c|c|}
\hline $\mathrm{CI}$ & Nivel Incompetencia & Nivel Competencia & & \\
\hline Conciencia & & Clase 2 & $\chi 2(3, \mathrm{~N}=250)=15,40 \mathrm{p}=.001$ & 0,241 \\
\hline Motivación & & & $\chi 2(3, N=253)=51,08 \mathrm{p} \leq .001$ & 0,410 \\
\hline Conocimiento & & Clase 1 & $\chi 2(3, \mathrm{~N}=252)=101,6 \mathrm{p} \leq .001$ & 0,536 \\
\hline Habilidades & Clase 4 & & $\chi 2(3, \mathrm{~N}=250)=81,80 \mathrm{p} \leq .001$ & 0,505 \\
\hline Encuentro & & Clase 2 & $\chi 2(3, N=251)=63,67 \mathrm{p} \leq .001$ & 0,450 \\
\hline TOTAL CI & & & $\chi 2(3, \mathrm{~N}=246)=110,25 \mathrm{p} \leq .001$ & 0,556 \\
\hline SI & Media & Elevada & & \\
\hline Implicación & & Clase 2 & $\chi 2(3, \mathrm{~N}=180)=17,41 \mathrm{p}=.001$ & 0,297 \\
\hline Respeto & & & $\chi 2(6, N=214)=13,19 \mathrm{p}=.040$ & 0,241 \\
\hline Confianza & & Clase 1 & $\chi 2(6, N=225)=52,44 \mathrm{p} \leq .001$ & 0,434 \\
\hline Disfrute & Clase 4 & & $\chi 2(6, \mathrm{~N}=232)=23,28 \mathrm{p}=.001$ & 0,302 \\
\hline Atención & & & $\chi 2(6, N=215)=47,25 \mathrm{p} \leq .001$ & 0,424 \\
\hline TOTAL SI & & Clase 1 & $\chi 2(6, \mathrm{~N}=249)=85,92 \mathrm{p} \leq .001$ & 0,506 \\
\hline
\end{tabular}

Tabla 7. Descriptivos por variables claves de análisis

\begin{tabular}{|c|c|c|c|c|c|}
\hline & & $\begin{array}{l}\text { Clase } 1 \\
(\mathrm{~N}=52)\end{array}$ & $\begin{array}{l}\text { Clase } 2 \\
(\mathrm{~N}=73)\end{array}$ & $\begin{array}{l}\text { Clase } 3 \\
(\mathrm{~N}=81)\end{array}$ & $\begin{array}{l}\text { Clase } 4 \\
(\mathrm{~N}=47)\end{array}$ \\
\hline \multirow{3}{*}{$\begin{array}{c}\text { Total } \\
\text { supuestos (\%) }\end{array}$} & Baja & 13,5 & 13,7 & 86,4 & 52,2 \\
\hline & Media & 61,5 & 76,7 & 12,3 & 43,5 \\
\hline & Alta & 25,0 & 9,6 & 1,2 & 4,3 \\
\hline \multicolumn{2}{|c|}{$\begin{array}{c}\text { Media supuestos adecuados } \\
(1-9)\end{array}$} & $5,13(\mathrm{SD}=1,80)$ & $4,78(S D=1,520)$ & $2,52(\mathrm{SD}=1,361)$ & $3,63(\mathrm{SD}=1,691)$ \\
\hline \multirow{6}{*}{$\begin{array}{c}\text { Escala } \\
\text { Intervención } \\
\text { (1-5 grado } \\
\text { acuerdo) }\end{array}$} & $\begin{array}{l}\text { Soy consciente } \\
\text { de mis sesgos }\end{array}$ & $2,00(\mathrm{SD}=0,950)$ & $2,33(\mathrm{SD}=0,852)$ & $2,00(\mathrm{SD}=0,920)$ & $2,07(\mathrm{SD}=0,848)$ \\
\hline & Habilidades & $2,96(\mathrm{SD}=0,196)$ & $2,50(\mathrm{SD}=0,654)$ & $2,51(\mathrm{SD}=0,636)$ & $2,04(\mathrm{SD}=0,796)$ \\
\hline & $\begin{array}{l}\text { Percibir la } \\
\text { diversidad }\end{array}$ & $2,92(\mathrm{SD}=0,272)$ & $2,23(\mathrm{SD}=0,685)$ & $2,29(\mathrm{SD}=0,715)$ & $1,80(\mathrm{SD}=0,734)$ \\
\hline & $\begin{array}{l}\text { Diseñar un } \\
\text { proyecto }\end{array}$ & $2,63(\mathrm{SD}=0,564)$ & $2,01(\mathrm{SD}=0,752)$ & $2,16(\mathrm{SD}=0,829)$ & $1,40(\mathrm{SD}=0,623)$ \\
\hline & $\begin{array}{l}\text { Conocimientos } \\
\text { intervención }\end{array}$ & $2,86(\mathrm{SD}=0,401)$ & $2,09(\mathrm{SD}=0,737)$ & $2,30(\mathrm{SD}=0,660)$ & $1,53(\mathrm{SD}=0,726)$ \\
\hline & Total escala & $3,82(\mathrm{SD}=0,545)$ & $3,26(\mathrm{SD}=0525)$ & $3,31(\mathrm{SD}=0,634)$ & $2,69(\mathrm{SD}=0,623)$ \\
\hline \multirow{6}{*}{$\begin{array}{c}\text { Competencia } \\
\text { Intercultural } \\
\quad(1-4)\end{array}$} & Conciencia & $2,30(\mathrm{SD}=1,015)$ & $2,71(\mathrm{SD}=0,874)$ & $2,26(\mathrm{SD}=1,010)$ & $1,96(\mathrm{SD}=0,868)$ \\
\hline & Motivación & $2,02(\mathrm{SD}=1,379)$ & $2,74(\mathrm{SD}=1,214)$ & $3,19(\mathrm{SD}=1,074)$ & $1,85(\mathrm{SD}=0,908)$ \\
\hline & Conocimiento & $3,06(\mathrm{SD}=0,366)$ & $2,86(\mathrm{SD}=0,484)$ & $2,83(\mathrm{SD}=0,495)$ & $2,04(\mathrm{SD}=0,415)$ \\
\hline & Habilidades & $3,24(\mathrm{SD}=0,555)$ & $3,07(\mathrm{SD}=0,561)$ & $3,08(\mathrm{SD}=0,652)$ & $2,21(\mathrm{SD}=0,690)$ \\
\hline & Encuentro & $3,18(\mathrm{SD}=0,800)$ & $3,03(\mathrm{SD}=0,707)$ & $3,00(\mathrm{SD}=0,822)$ & $2,11(\mathrm{SD}=0,759)$ \\
\hline & Total CI & $13,96(\mathrm{SD}=2,259)$ & $14,42(\mathrm{SD}=1,750)$ & $14,33(\mathrm{SD}=1,868)$ & $10,13(S D=2,146)$ \\
\hline
\end{tabular}




\begin{tabular}{cccccc}
\hline & Implicación & $4,38(\mathrm{SD}=0,514)$ & $4,20(\mathrm{SD}=0,506)$ & $4,15(\mathrm{SD}=0,461)$ & $3,72(\mathrm{SD}=0,611)$ \\
Sensibilidad & Respeto & $4,59(\mathrm{SD}=0,613)$ & $4,54(\mathrm{SD}=0,391)$ & $4,58(\mathrm{SD}=0,386)$ & $4,12(\mathrm{SD}=0,779)$ \\
$\begin{array}{c}\text { Intercultural } \\
(1-5)\end{array}$ & Confianza & $4,08(\mathrm{SD}=0,505)$ & $3,56(\mathrm{SD}=0,574)$ & $3,74(\mathrm{SD}=0,536)$ & $3,20(\mathrm{SD}=0,645)$ \\
& Disfrute & $4,37(\mathrm{SD}=0,864)$ & $4,14(\mathrm{SD}=0,841)$ & $4,14(\mathrm{SD}=0,824)$ & $3,57(\mathrm{SD}=0,885)$ \\
& Atención & $4,16(\mathrm{SD}=0,778)$ & $4,16(\mathrm{SD}=0,606)$ & $4,06(\mathrm{SD}=0,514)$ & $3,27(\mathrm{SD}=0,553)$ \\
& Total escala & $4,32(\mathrm{SD}=0,534)$ & $4,14(\mathrm{SD}=0,329)$ & $4,17(\mathrm{SD}=0,293)$ & $3,69(\mathrm{SD}=0,417)$ \\
\hline
\end{tabular}

\section{Discusión y conclusiones}

Los resultados ponen de manifiesto algunas asociaciones significativas entre las respuestas a los supuestos planteados y las variables claves del estudio.

En relación a la caracterización no se producen diferencias en el número de supuestos respondidos adecuadamente debido a pertenecer a una u otra profesión de las que conforman los equipos base de los Servicios Sociales Comunitarios. La inexistencia de asociaciones representativas con ciertas variables sociodemográficas pone de manifiesto que las características personales/ profesionales no influyen significativamente en las respuestas.

Sin embargo, el hecho de ser un profesional de mayor edad (entre los $50 \mathrm{y}$ 64 años) se relaciona con responder inadecuadamente a los supuestos cuarto y quinto, reflejando menor flexibilidad cognitiva, empatía y menor comprensión de aspectos de otras culturas diferentes a la propia. Esto puede estar relacionado con un menor acceso durante su trayectoria profesional a cuestiones formativas y de sensibilización con respecto a la diversidad cultural, entre otros aspectos.

Hay otras cuestiones de caracterización personal que destacan como tener formación en diversidad y mantener contacto con personas de origen extranjero, ya que ambas se asocian con responder adecuadamente al supuesto sexto relacionado con mayor habilidad para formar y mantener relaciones y con el mantenimiento de la distancia social adecuada, algo que sin duda se favorece cuando el profesional dispone de formación específica sobre la diversidad cultural y cuando además mantiene contacto con personas de culturas diferentes a la propia, propiciando la familiarización y el aprendizaje en el manejo de las relaciones sociales, aspectos vinculados con la primera de las clases extraídas. El contacto también propicia respuestas adecuadas al supuesto séptimo (en el que el profesional insiste en los derechos al usuario), que muestra las habilidades de conocimiento y comprensión tanto de la propia cultura como de otras culturas diferentes, que de nuevo se asocia con pertenecer a la primera clase.

Poseer un cierto nivel de CI se asocia, en ciertos casos, con resolver con acierto las intervenciones profesionales. Cabe destacar que son los supuestos segundo y octavo los que tienen asociaciones en este sentido. Concretamente el segundo supuesto que refleja el conocer y comprender tanto la propia cultura 
como otras, se relaciona con niveles de CI elevados, sobre todo de conciencia y de conocimiento, así como con una elevada implicación de la SI y con pertenecer a la segunda de las cuatro clases extraídas.

El no saber mantener la distancia social apropiada (supuesto octavo) está asociado con una menor disponibilidad al encuentro intercultural, así como menor habilidad de gestión de la diversidad en la intervención profesional. Aquellos profesionales que sí disponen de buenas respuestas a esta supuesto, y por ello se ubican en la clase primera, con niveles elevados de competencia en el encuentro y en las habilidades mencionadas.

El sexto supuesto también refleja la competencia de saber manejar adecuadamente la distancia social en las interacciones y se relaciona con disponer de formación en diversidad y con mantener contacto con personas extranjeras, variables claves para el desarrollo de la competencia intercultural.

Responder adecuadamente a mayor número de supuestos se asocia con aquellas personas que tienen mayor conciencia intercultural, mayor motivación por el encuentro. Esta mayor motivación también conlleva una adecuada respuesta al supuesto primero donde se refleja la habilidad para no enjuiciar siendo consciente de las diferencias culturales existentes. Es la clase primera la que aglutina el mayor número de supuestos adecuadamente resueltos, que se asocia a una mayor conciencia de los propios sesgos y prejuicios posibles hacia las diferencias culturales (como aspecto de intervención).

Las clases extraídas han puesto de manifiesto la existencia de aspectos claves para disponer de respuestas adecuadas a los supuestos y que se relacionan con niveles de $\mathrm{CI}$ elevados en las diferentes dimensiones contempladas, así como niveles elevados de SI que permiten disponer de aspectos claves para el desarrollo de la praxis profesional en contextos de diversidad. Junto a estas cuestiones también aparece asociado el hecho de tener contacto con personas extranjeras y formación en diversidad o conocimiento de idiomas. La neutralidad, correspondida por la clase tercera de la tipología, se ubica en niveles bajos de respuestas adecuadas a los supuestos y una indiferencia en cuestiones de intervención fundamentalmente.

Las competencias asociadas a estos supuestos son bastante relevantes en el desempeño profesional en contextos de diversidad cultural. El hecho de que la formación específica y el contacto con personas de culturas diferentes sea relevante en la asociación con responder de un modo adecuado a ciertos supuestos, pone de manifiesto que se trata de cuestiones claves a considerar en estas profesiones y en el propio reciclaje y formación continua de los profesionales de los Servicios Sociales Comunitarios. Esta formación y el contacto son elementos diferenciadores en la práctica y en cuestiones que tienen que 
ver con la interacción directa con el otro (motivación y habilidades). Trabajar la formación en diversidad e interculturalidad de manera transversal permitirá aumentar conocimientos, habilidades, actitudes que, unido a lo positivo que proporciona el contacto con personas extranjeras, permitirá un mejor y más adecuado desarrollo profesional en contextos de diversidad cultural.

Resulta por lo tanto más relevante, y al mismo tiempo se desarrollan de un modo más adecuado, cuestiones que tienen que ver con el conocimiento y la comprensión de la propia cultura y de aspectos de otras culturas, así como la habilidad de no enjuiciar, saber tener relaciones con personas de diversas culturas y, al mismo tiempo, mantener las distancia social adecuada en cada momento (y que son los supuestos que más asociaciones y relevancia han adquirido en los resultados obtenidos). Estos aspectos están relacionados con competencias y habilidades interculturales que tienen los profesionales de los Servicios Sociales Comunitarios como la conciencia intercultural, la motivación por el encuentro y el propio desarrollo de las interacciones. De igual modo, destaca la implicación de la SI en la gestión y la comprensión de las diferencias y características de personas y situaciones de otras culturas diferentes a la propia, manejando y siendo conscientes de los propios sesgos personales.

En este sentido, y siguiendo las recomendaciones de la American Psychological Association (2003), se puede trabajar en cuanto a los profesionales de la intervención social el mantenimiento y mejora del conocimiento de las propias creencias, valores, actitudes, realizando un esfuerzo por el cambio de las percepciones automáticas positivas para el endogrupo y negativas para el exogrupo, pudiendo cambiar sesgos y prejuicios. Habría que trabajar cuestiones que están menos desarrolladas y/o afianzadas como la tolerancia a la ambigüedad en situaciones profesionales complejas donde está presente la diversidad cultural, reforzar la flexibilidad cognitiva, la empatía y la habilidad de resolución de conflictos en estas situaciones y con personas usuarias de diferentes culturas.

Estas cuestiones pueden y deben ser trabajadas con los profesionales de los Servicios Sociales Comunitarios, con todas las profesiones presentes dado que no se han hallado diferencias relevantes en función de la profesión. Se pueden elaborar propuestas de formación encaminadas a mejorar y adecuar los servicios y la propia CI profesional.

Pero además de realizar este trabajo de formación y sensibilización profesional continua, habría que incidir en las etapas de formación universitaria. Las Facultades de las diferentes profesiones deben potenciar el desarrollo de competencias interculturales específicas y transversales. El ámbito universitario debe propiciar la reflexión y la reformulación de la definición de CI, reconociendo su carácter complejo, dinámico, interseccional los elementos de poder 
y las macro estructuras implicadas además de los elementos individuales y comunitarios culturales presentes en las situaciones de atención e intervención profesional.

\section{Bibliografía}

American Psychological Association (2010). Ethical Principles of Psychologists and Code of Conduct. United States: American Psychological Association.

Asociación Estatal de Educación Social (2004). Código deontológico del educador y la educadora social. Barcelona, España: Asociación Estatal de Educación Social.

BalCaZAR, F. FABricio, SuARez-BAlCAZAR, M. y Willis, E. (2010). Cultural competence: A review of conceptual frameworks. En Balcazar, Suarez-Balcazar, Taylor-Ritzler and Keys (Eds.), Race, culture, and disability: Rehabilitation science and practice (pp.281-305). Boston, EEUU: Jones \& Bartlett.

Berry, J. V., Pootinga, Y., Segall, M. y Dasen, P. (2002). Cross-cultural Psychology. Research and applications. New York, EEUU: Cambridge University Press.

COHEN, J. (1988). Statistical power analysis for the behavioral sciences. Nueva York, EEUU: Academic Press. https://doi.org/10.1002/bs.3830330104

Consejo General del Trabajo Social (2012). El Código Deontológico del Trabajo Social. Madrid, España: Consejo General del Trabajo Social.

DANA, R. AND ALLEN, J. (2008). Cultural competency training in a global society. New York, EEUU: Springer. https://doi.org/10.1007/978-0-387-79822-6

DomeneCh-Rodríguez, M. M., Baumann, A. y SChwartZ, A. (2011). Cultural Adaptation of an Evidence Based Intervention: From Theory to Practice in a Latino/a Community Context. American Journal Community Psychology, 47, 170-186. https://doi.org/10.1007/s10464-010-9371-4

Dumas, J. E., Rollock, D., Prinz, R., Hops, H. y Blechman, E. (1999). Cultural sensitivity: Problems and solutions in applied and preventive intervention. Applied \& Preventive Psychology, 8, 175-196. https://doi.org/10.1016/ S0962-1849(05)80076-9

GARRAN, A. M. y WeRKMEISTER, L. (2013). Cultural Competence Revisited. Journal of Ethnic \& Cultural Diversity in Social Work, 22 (97), 111-121. https://doi.org /10.1080/15313204.2013.785337

GENG, C. y AwA, N. (1992). Measuring intercultural effectiveness: An integrative approach. International Journal of Intercultural Relations, 16, 311-328. https:// doi.org/10.1016/0147-1767(92)90055-Y

Guo-Ming, C. y Starosta, W. (2000). The development and validation of the intercultural sensitivity scale. Human Communication, 3, 1-15.

instituto Nacional de Estadística de España (2013). Población por lugar de nacimiento. Madrid, España: INE. Gobierno de España. 
INTERNATIONAL FEDERATION OF SOCIAL WORKERS (2004). Statement of ethical principles. Recuperado de http://ifsw.org/policies/statement-of-ethical-principles/

INTERNATIONAL ASSOCIATION OF SCHOOLS OF SOCIAL WORK (2004). Ethics in social work, statement of principles. Australia: Adelaide. Recuperado de http://www. ifsw.org/f38000032.html

Julve, M. y PAlOMO, B. (2005). La competencia comunicativa intercultural en la prestación de servicios. Glosas didácticas-Revista electrónica internacional, 15, 26-38.

Kohli, H. K., Huber, R. y FAUL, A. (2010). Historical and Theoretical Development of Culturally Competent Social Work Practice. Journal of Teaching in Social Work, 30 (3), 252-271. https://doi.org/10.1080/08841233.2010.499091

Martínez, M.F., Martínez, J. y CAlzado, V. (2006). La Competencia Cultural como referente de la Diversidad Humana en la Prestación de Servicios y la Intervención Social. Intervención Psicosocial, 15(3), 331-350. https://doi. org/10.4321/S1132-05592006000300007

MAYA, I. (2002). Estrategias de entrenamiento de las habilidades de comunicación intercultural. Portularia, 2, 91-108.

NATIONAL ASSOCIATION OF SOCIAL WORKERS (2001). Standards for cultural competence in social work practice. Recuperado de http://www.naswdc.org/ practice/ standards/NAswculturalstandards.pdf

Skelton, T. y Allen, T. (1999). Culture and global change. London, UK: Routledge.

Ungar, M. (2002). Deeper, More Social Ecological Social Work Practice. Social Service Review, 76(3), 480-497. https://doi.org/10.1086/341185

VÁzquez-Aguado, O., Fernández, M., y FernándeZ-Borrero, M.A. (2010). Good intercultural practices in social services projects. Journal of Social Service Research, 36(4), 303-320. https://doi.org/10.1080/01488376.2010.493853

VÁzquez-Aguado, O., Fernández-Borrero, M.A., y Álvarez-PÉrez, P. (2014). La aportación de los grados al desarrollo de la sensibilidad y la competencia intercultural. Perspectiva comparada entre Trabajo Social y Psicología. Cuadernos de Trabajo Social, 27(2), 307-317. https://doi.org/10.5209/rev_CUTS.2014. v27.n2.44679

VILÁ, R. (2005). La Competencia Comunicativa Intercultural. Un estudio en el Primer Ciclo de la E.S.O. Tesis Doctoral. Barcelona, España: Universidad de Barcelona.

Wiseman, R. L., HAMmer, M. y NishidA, H. (1989). Predictors of intercultural communication competence. International Journal of Intercultural Relations, 13, 349-370. https://doi.org/10.1016/0147-1767(89)90017-5

\section{ANTECEDENTES DE DIFUSIÓN}

La difusión de los resultados del presente estudio ha contado con el apoyo del Ministerio de Educación, Cultura y Deporte del Gobierno de España a través del Programa Nacional de Formación de Profesorado Universitario (FPU15/03909) de la tercera autora. 